NBSIR 85-3206

\title{
Global Solutions to Factorable Nonlinear Optimization Problems Using Separable Programming Techniques
}

Garth P. McCormick

U.S. DEPARTMENT OF COMMERCE

National Bureau of Standards

National Engineering Laboratory Center for Applied Mathematics Gaithersburg, MD 20899

June 1985

Sponsored by

U.S. DEPARTMENT OF COMMERCE

National Bureau of Standards

$-Q C \longrightarrow$ Applied Mathematics

100

is Research Division

.456

Jurg, MD 20899

$85-3206$ 
NBSIR 85-3206

...

GLOBAL SOLUTIONS TO FACTORABLE

NONLINEAR OPTIMIZATION PROBLEMS

USING SEPARABLE PROGRAMMING

TECHNIQUES

Garth P. McCormick

U.S. DEPARTMENT OF COMMERCE

National Bureau of Standards

National Engineering Laboratory

Center for Applied Mathematics

Gaithersburg, MD 20899

June 1985

Sponsored by

U.S. DEPARTMENT OF COMMERCE

National Bureau of Standards

Center for Applied Mathematics

Operations Research Division

Gaithersburg, MD 20899

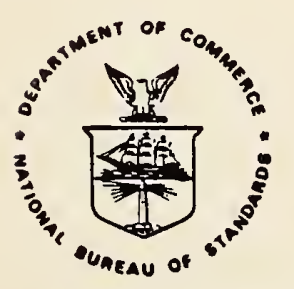

U.S. DEPARTMENT OF COMMERCE, Malcolm Baldrige, Secretary

NATIONAL BUREAU OF STANDARDS, Ernest Ambler, Director 

ABSTRACT

Many algorithms for obtaining global solutions to nonconvex optimization problems have been proposed in recent years. The methods farthest along computationally are those for separable problems. These use linear programming codes to solve sequences of LP problems formed from piece-wise linear approximations to the nonlinear functional forms. For a large class of optimization problems, called factorable programming problems, it is possible to create equivalent separable problems. This is done at a cost: additional variables and constraints. In this paper the procedure for creating the equivalent separable problems is outlined and a brief description is given of a global solution algorithm due to Falk. A small example is given illustrating the above techniques. The example is also solved using a more direct method. Application to the solution of nonlinear least squares is illustrated with another example. Discussion of areas of research for improving the efficiency of this approach concludes the paper. 


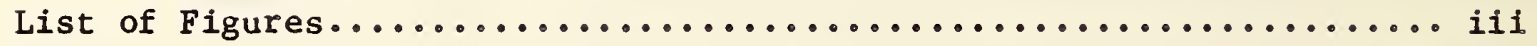

List of Tables................................ iv

1. Global Solutions to Separable Problems..................... 1

2. Factorable Programing Problems....................... 5

3. Creating Equivalent Separable Programs................... 8

4. Obtaining Bounds on the New Variables.................... 13

5. Computer Solution to Simple Problems..................... 15

6. Direct Solution of Simple Problems...................... 18

7. Application to Nonlinear Least Squares.................... 26

8. Discussion of Results and Areas of Future Research.......... 35

9. $\operatorname{Ref} e r e n c e s . \ldots \ldots \ldots \ldots \ldots \ldots \ldots \ldots \ldots \ldots \ldots \ldots \ldots \ldots \ldots \ldots \ldots$

Appendix A Listings of Subroutine GETPHI and Data Input File for

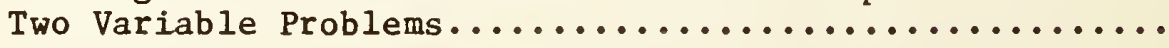

Appendix B Listings of Subroutines and Data File for Least Squares

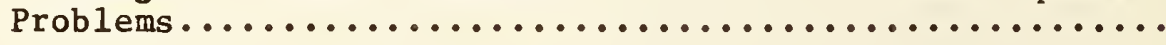




\section{LIST OF FIGURES}

Page

Figure 1 - Piecewise Linear Approximations.................... 3

Figure 2 - Isovalue Contours of Two Variable Problem............. 7

Figure 3 - Convex Envelope of Sine Function in $[0,10] \ldots \ldots \ldots$

Figure 4 - A Convex Underestimating Function.................. 22

Figure 5 - Isovalue Contours of Convex Underestimating Function..... 25

Figure 6 - Plotted Data for Example........................ 29 
Table 1 - Computer Effort Required to Solve LP Approximating

Problems................................... 17

Table 2 - Data for Example............................ 28

Table 3 - Data for Least Squares Problem in Separable Form......... 32

Table 4 - Data Relating to Solution of Least Squares Problem........ 34 


\section{Global Solutions to Separable Problems}

Solving nonconvex programming problems, i.e., optimization problems where local minimizers may not be global minimizers was thought not long ago to be relegated to heuristic algorithms. (See [McCormick 1972a] for a survey of some of these methods.) Recent investigators (e.g. [Falk and Soland 1969], [Soland 1971], [Falk 1972], [Beale and Tomlin 1970], [Hoffman 1981], [Mancini and McCormick 1976], [Mancini and McCormick 1979], [McCormick 1980]) have developed rigorous algorithms for which convergence to global minimizers can be proved. The theories are well-established and some computational results are available.

The algorithms which have the most computational development are those which solve separable optimization problems using linear programming codes. Separable programming problems have the following form:

$$
\begin{aligned}
& \operatorname{minimize} F_{o}(x) \\
& \quad x \in R n \\
& \text { sub ject to } F_{i}(x) \leqslant b_{i}, i=1, \ldots, m, \\
& \qquad \ell_{j} \leqslant x_{j} \leqslant L_{j}, j=1, \ldots, n,
\end{aligned}
$$

where each $F_{i}(x)$ is written

$$
F_{i}(x)=\sum_{j=1}^{n} F_{i j}\left(x_{j}\right), i=0,1, \ldots, m .
$$

(A more general formulation allows for equality constraints: i.e. for $F_{i}(x)=b_{i}, i=m+1, \ldots, p$. However, for simplicity in presentation only, the inequality constrained problem is considered in this section.) 
The basic idea behind most of these methods is to approximate each nonlinear functional by a piece-wise linear functional and solve the resulting problem by solving a finite sequence of linear programming problems. The computer programs used to solve the example of this paper implemented the algorithm of Falk and is briefly described below. For more details the reader is referred to [Falk 1972] or Appendix A in [Grotte 1978].

The approximating problem of the original problem $Q$ is obtained by replacing each function $F_{i j}$ by a piece-wise linear approximation over the interval $\left[\ell_{j}, L_{j}\right]$. This involves selecting $p_{j}+1$ grid points $y_{j o}, \ldots, y_{j p_{j}}$ in $\left[\ell_{j}, L_{j}\right]$, where $y_{j o}=\ell_{j}$ and $y_{j p_{j}}=L_{j}$. Suppose $x_{j *}$ is some point in $\left[\ell_{j}, L_{j}\right]$. Let $y_{j k *}, y_{j k *+1}, \Theta_{y k^{*}}$ be the unique values where

$$
x_{j *}=\theta_{j k *} y_{j k^{*}}+\left(1-\theta_{j k^{*}}\right) y_{j k^{*}+1}, 0<\theta_{j k^{*}} \leqslant 1
$$

Then

$$
F_{i j}\left(x_{j *}\right)=\theta_{j k^{*}} F_{i j}\left(y_{j k^{*}}\right)+\left(1-\Theta_{j k^{*}}\right) F_{i j}\left(y_{j k^{*}+1}\right) \text {. }
$$

Figure 1 represents this type of approximation.

There are obviously many ways these $\mathrm{pj}_{\mathrm{j}}+1$ points can be selected. The computer program implementing this algorithm allows the user to specify the value $p_{j}$. It then computes equally spaced points.

Let $\mathrm{K}_{\mathrm{j}}=\left\{0,1, \ldots, \mathrm{p}_{\mathrm{j}}\right\}$. The approximating problem can be stated in terms of the weights on the grid points, the $\theta_{j k}$ 's: 


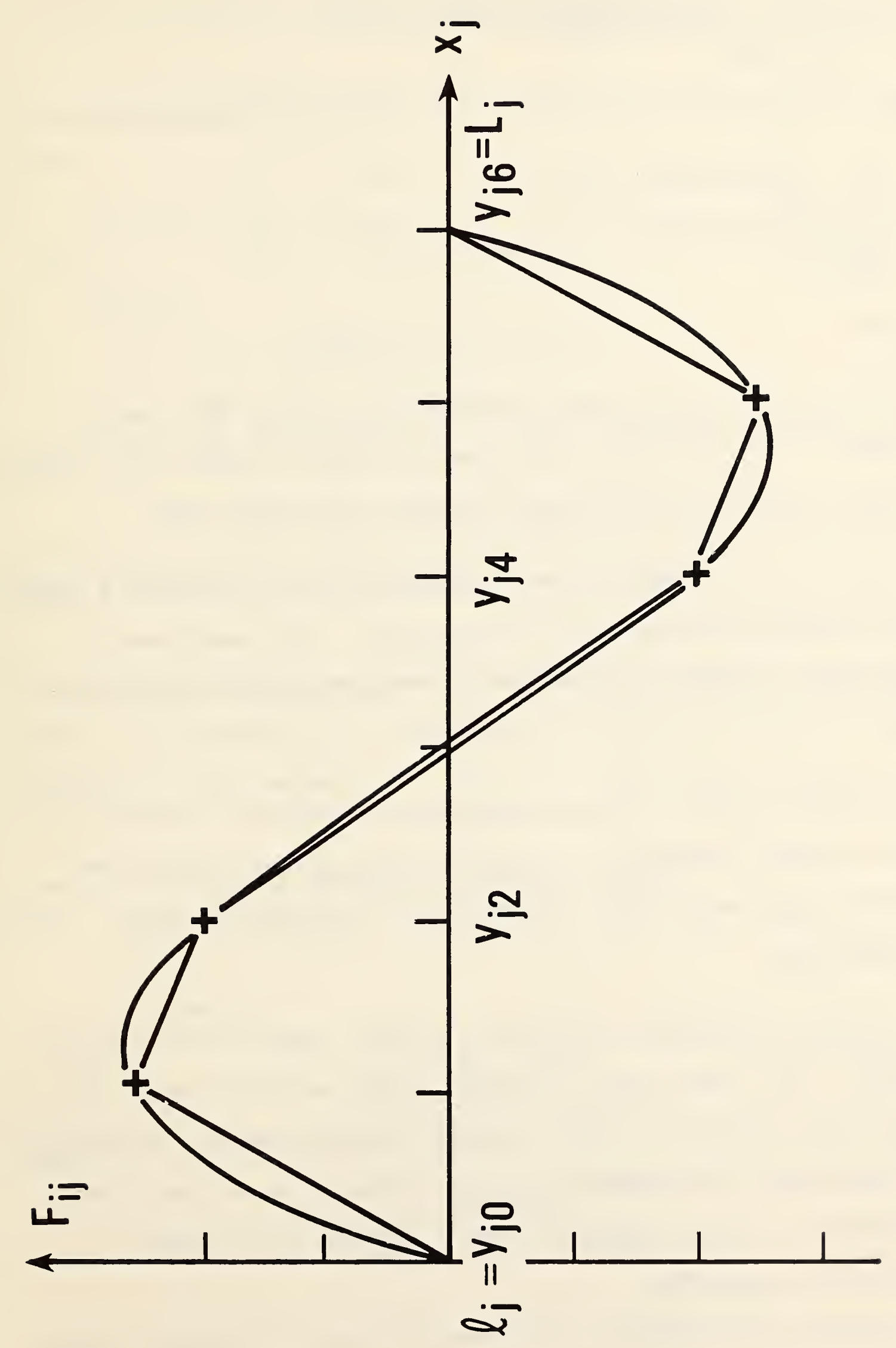




$$
\min _{\theta} \sum_{j=1}^{n} \sum_{k \varepsilon} K_{j} \Theta_{j k} F_{o j}\left(y_{j k}\right)
$$

subject to

$$
\begin{aligned}
& \sum_{j=1}^{n} \sum_{k \varepsilon K_{j}} \theta_{j k} F_{i j}\left(y_{j k}\right)<b_{i}, i=1, \ldots, m, \\
& \sum_{k \varepsilon K_{j} \theta_{j k}}=1 \quad j=1, \ldots, n, \\
& \theta_{j k} \\
& >0, j=1, \ldots, n ; k \varepsilon k_{j}
\end{aligned}
$$

and (for $j=1, \ldots, n$ ) at most two of the weights $\left\{\Theta_{j k}, k \varepsilon k_{j}\right\}$ can be nonzeros. If two are nonzero, they must correspond to adjacent grid points. This last constraint is the adjacency weighted restriction (AWR).

If it were not for the AWR, the approximating problem (P) would be a simple linear programming problem with variables $\left\{\Theta_{j k}\right\}^{*}$. This restriction is necessary in order that the piecewise linear approximation indicated be valid.

In [Falk 1972] a branch and bound algorithm for solving ( $P$ ) is presented. It involves solving a sequence of linear programming problems without the adjacency weight restrictions. The details of his algorithm however, will not be pursued here.

Most nonlinear programming problems are, in their simplest formulation, not separable. For a large class of problems, called factorable programming problems it is possible to create separable programs which are "equivalent" to the original ones in the sense that local minimizers are in a one-to-one correspondence. This is done at a cost; an increase in the number of variables and constraints.

The description of factorable optimization problems is in the next section. 
A factorable programming problem is one which can be written in the following form:

$\min f_{N}(x) \equiv \sum_{p=1}^{N-1} T_{N, p}\left[f_{p}(x)\right]+\sum_{p=1}^{N-1} \sum_{q=1}^{p} U_{N, p, q}\left[f_{q}(x)\right] \cdot v_{N, q, p}\left[f_{p}(x)\right]$ sub ject to

$$
a_{j} \leqslant f_{j}(x) \equiv x_{j}<b_{j}, j=1, \ldots, n,
$$

$a_{j} \leqslant f_{j}(x) \equiv \sum_{p=1}^{j-1} T_{j, p}\left[f_{p}(x)\right]+\sum_{p=1}^{j-1} \sum_{q=1}^{p} U_{j, p}, q,\left[f_{q}(x)\right] v_{j, q, p}\left[f_{p}(x)\right] \leqslant b_{j}$, $j=m+1, \ldots, N-1$ where some of the $a_{j}$ 's are possibly equal to $-\infty$, and the $T^{\prime} s, U^{\prime} s$ and $V^{\prime} s$ are scalar functions of one variable.

Most practical nonlinear programming problems are factorable. The reader is referred to [McCormick 1975] and [Ghaemi and McCormick 1979] for a fuller discussion of this subject.

Consider the following nonlinear programming problem:

$$
\begin{aligned}
& \underset{\min }{\left(x_{1}, x_{2}\right)} \text { ERF }\left(x_{1}+x_{2}\right)+\sin \left(x_{1}\right) \cdot \exp \left(-.5 x_{2}\right) \\
& \text { subject to } \\
& \\
& \quad-\left(x_{1}+x_{2}\right)^{2} \leqslant-10 \\
& 0 \leqslant x_{1} \leqslant 10,0 \leqslant x_{2} \leqslant 10,
\end{aligned}
$$

where

$$
\begin{gathered}
k=2 / \sqrt{ }, \\
\operatorname{ERF}(z)=k \int_{0}^{z} \exp \left(-t^{2}\right) d t
\end{gathered}
$$


Written as a factorable programming problem $f_{j}=f_{j}(x)$ ) this has the form:

$$
\underset{\left(x_{1}, x_{2}\right)}{\min } f_{5}=\operatorname{ERF}\left(f_{3}\right)+\sin \left(f_{1}\right) \cdot \exp \left(-.5 f_{2}\right)
$$

subject to

$$
\begin{aligned}
& 0<\mathrm{f}_{1} \equiv \mathrm{x}_{1}<10 \\
& 0<\mathrm{f}_{2} \equiv \mathrm{x}_{2}<10 \\
& -\infty<\mathrm{f}_{3} \equiv \mathrm{f}_{1}+\mathrm{f}_{2}<+\infty \\
& -\infty<\mathrm{f}_{4} \equiv-\left(\mathrm{f}_{3}\right)^{2} \leqslant-10
\end{aligned}
$$

The isovalue contours of the objective function are plotted in Figure 2 . There are two local minimizers, one at $(10,0)$ with objective function value .45599 , and another at approximately $(3 \pi / 2,0)$ with objective function value approximately 0 . If the inequality constraint $\left(x_{1}+x_{2}\right)^{2} \geqslant 10$ were removed from the problem, $(0,0)$ would also be a local minimizer. The point $(3 \pi / 2,0)$ is the global minimizer in both cases with a function value slightly less than zero. 


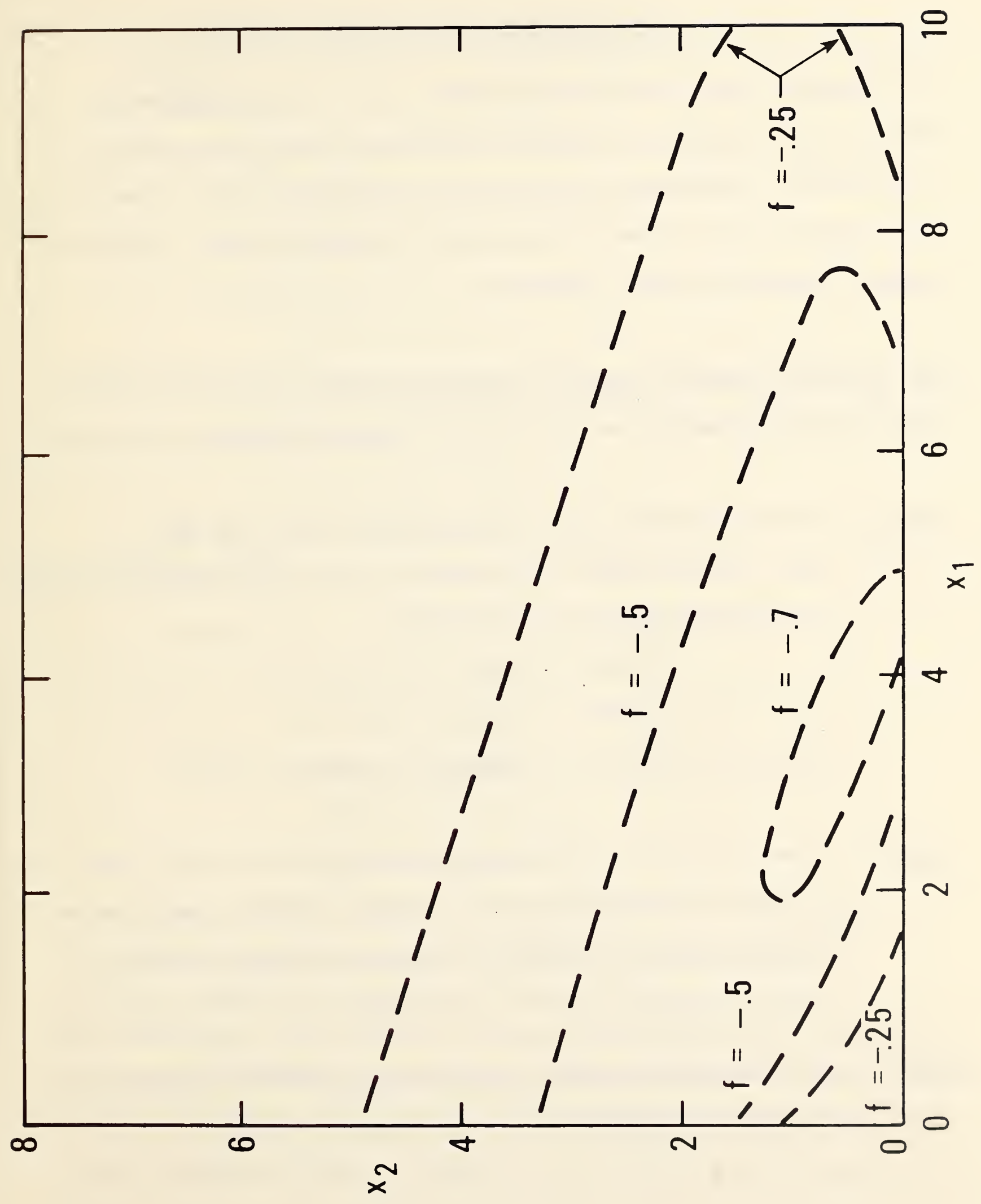

Figure 2. Isovalue Contours of Two Variable Problem 


\section{Creating Equivalent Separable Problems}

The techniques which can be used to convert most nonlinear programming problems into "equivalent" separable programs are part of the tradition of optimization. The origins of these simple techniques are lost in the past. A description of two of these are contained in [McCormick 1972b] . Below is a summary statement of these techniques.

The method for creating equivalent separable problems has two basic steps which are used repeatedly until they can no longer be applied.

Step 1. If the optimization poblem has a product term of the form $q_{1}(x) \cdot q_{2}(x)$, replace that product by $\left(z_{1}\right)^{2}-\left(z_{2}\right)^{2}$ wherever it occurs, and introduce the equality constraints

$$
\begin{aligned}
& q_{1}(x)=z_{1}+z_{2} \\
& q_{2}(x)=z_{1}-z_{2} .
\end{aligned}
$$

The problem variables are augmented to include $z_{1}$ and $z_{2}$.

Step 2. If the optimization problem has a term of the form $T[t(x)]$ where $T(t)$ is a scalar function and $t(x)$ is a scalar function of more than one variable, replace $T[t(X)]$ by $T(y)$ wherever it occurs, add the equality constraint $t(x)=y$, and augment the variable list with $y$.

Consider the example given earlier. Applying step 2 for the expression $t(x)=x_{1}+x_{2}$ yields the program 


$$
\begin{aligned}
& \min \operatorname{ERF}\left(y_{1}\right)+\sin \left(x_{1}\right) \cdot \exp \left(-.5 x_{2}\right) \\
& \left(x_{1}, x_{2}, y_{1}\right) \\
& \text { sub ject to }
\end{aligned}
$$

$$
\begin{aligned}
& -\left(y_{1}\right)^{2}<-10 \\
& x_{1}+x_{2}=y_{1} \\
& 0<x_{1}<10 \\
& 0<x_{2}<10
\end{aligned}
$$

Application of Step 1 to the product term in the objective function yields the equivalent separable program

$$
\begin{aligned}
& \min \operatorname{ERF}\left(y_{1}\right)+\left(z_{1}\right)^{2}-\left(z_{2}\right)^{2} \\
& \left(x_{1}, x_{2}, y_{1}, z_{1}, z_{2}\right)
\end{aligned}
$$

sub ject to

$$
\begin{aligned}
& -\left(y_{1}\right)^{2} \leqslant-10 \\
& x_{1}+x_{2}=y_{1}, \\
& \sin \left(x_{1}\right)=z_{1}+z_{2}, \\
& \exp \left(-.5 x_{2}\right)=z_{1}-z_{2}, \\
& 0 \leqslant x_{1} \leqslant 10,0<x_{2} \leqslant 10 .
\end{aligned}
$$

For this simple problem, separation occurred after just two applications of the separating techniques. Often the situation is more complicated.

The "equivalence" of the problems is shown in [McCormick 1972c], where it is shown that local minimizers are in one-to-one correspondence. For the purpose here it is sufficient to emphasize that if $\left(x_{1}, x_{2}, y_{1}, z_{1}, z_{2}\right)$ is a local (global) minimizer for (3.2), then $\left(\mathrm{x}_{1}, \mathrm{x}_{2}\right)$ is a local (global) minimizer for $(3.1)$ 
Not all nonlinear programming problems can be separated by repeated application of steps 1 and 2. In particular, if a program cannot be written as a factorable optimization problem it probably cannot be separated. Certainly, if it can be written in factorable form, separation is possible.

Make the correspondence $y_{j}=x_{j}, j=1, \ldots, m$. Then using steps 1 and 2 on the factorable programming problem (2.1) yields directly

$$
\min _{(y, z)} \sum_{p=1}^{N-1} T_{N, p}\left(y_{n}\right)+\sum_{p=1}^{N-1} \sum_{q=1}^{p}\left[\left(z_{q, p}^{N, 1}\right)^{2}-(z q, p)^{2}\right]
$$

subject to the inequality constraints

$$
a_{j}<y_{j}<b_{j}, j=1, \ldots, N-1
$$

and the equality constraints

$$
\begin{array}{ll}
y_{j}=\sum_{p=1}^{j-1} T_{j, p}\left(y_{p}\right)+\sum_{p=1}^{j-1} \sum_{q=1}^{p}\left[\left(z_{q, p}^{j, 1}\right)^{2}-\left(z_{q, p}^{j, 2}\right)^{2}\right], j=m+1, \ldots, N-1 ; \\
U_{j, p, q}\left(y_{q}\right)=z_{q, p}^{j, 1}+z_{q, p}^{j, 2} & j=n+1, \ldots, N ; \\
v_{j, p, q}\left(y_{p}\right)=z_{q, p}^{j, 1}-z_{q, p}^{j, 2} & p=1, \ldots, j-1,
\end{array}
$$

Here $y=\left(x_{1}, \ldots, x_{m}, y_{m+1}, \ldots, y_{N-1}\right)^{T}$, and $z$ is the vector containing the $\mathrm{z}_{\mathrm{q}, \mathrm{p}}^{\mathrm{j}, 1}, \mathrm{z}_{\mathrm{q}, \mathrm{p}}^{\mathrm{j}, 2}$ 's appearing above.

Application of these equations to the example problem given in form (2.3) yields the separable problem 


$$
\min \operatorname{ERF}\left(y_{3}\right)+\left(z_{1,2}^{5,1}\right)^{2}-\left(z_{1,2}^{5,2}\right)^{2}
$$

sub ject to

$$
\begin{aligned}
& 0<y_{1}<10 \\
& 0<y_{2}<10 \\
& -\infty<y_{3}<+\infty \\
& -\infty<y_{4}<+\infty \\
& y_{3}=y_{1}+y_{2}, \\
& y_{4}=\left(y_{3}\right)^{2} \\
& \sin \left(y_{1}\right)=z_{1,2}^{5,1}+z_{1,2}^{5,2} \\
& \exp \left(-.5 y_{2}\right)=z_{1,2}^{5,1}-z_{1,2}^{5,2},
\end{aligned}
$$

where the problem variables are

$$
\left(y_{1}, y_{2}, y_{3}, y_{4}, z_{1,2}^{5,1}, z_{1,2}^{5,2}\right)
$$

Problem (3.8) is similar to the separable problem (3.2) obtained directly by applying steps 1 and 2 except that it has one variable more and one more equality constraint. This is an example of a general modification which should be made to the separation rules based to create (3.3). If some $f_{j}$ $(j=m+1, \ldots, N-1)$ does not enter into the computation of any future $f_{i}$, the introduction of the new variable $y_{j}$ should be omitted, and no equality constraint of the form (3.5) should be included. The simple bound in (3.4) in this instance should remain

$$
a_{j}<\sum_{p=1}^{j-1} T_{j, p}\left(y_{p}\right)+\sum_{p=1}^{j-1} \sum_{q=1}^{p}\left[\left(z_{q, p}^{j, 1}\right)^{2}-\left(z_{q, p}^{j, 2}\right)^{2}\right]<b_{j} .
$$


Using this modification, problem (3.8) above would be changed in that $\mathrm{y}_{4}$ would no longer be a variable, (3.10) would be omitted, and (3.9) would become $-\infty \leqslant-\left(y_{3}\right)^{2} \leqslant-10$. It is then equivalent, except for the variable names, to problem (3.2). 


\section{Obtaining Bounds on the New Variables}

Algorithms which obtain global solutions to separable optimization problems invariably require upper and lower bounds on the variables. (An inductive process to do this when all original variables $\left\{x_{j}\right\}$ are bounded is given below).

The basic assumption here is that it is possible to compute the suprema and infima of T's, U's, and $V^{\prime}$ s used in defining the factorable programming problem (2.1).

Define

$$
\begin{aligned}
& \ell(T, p, j)=\inf T_{j, p}(y) \text { s.t. } a_{p}<y<b_{p}, \\
& u(T, p, j)=\sup T_{j, p}(y) \text { s.t. } a_{p} \leqslant y \leqslant b_{p}, \\
& \ell(U, p, q, j)=\inf U_{j, p, q}(y) \text { s.t. } a_{q} \leqslant y \leqslant b_{q}, \\
& u(U, p, q, j)=\sup U_{j, p, q}(y) s \cdot t \cdot a_{q} \leqslant y \leqslant b_{q} \cdot
\end{aligned}
$$

Analagous notation is used for the other quantities.

Then the lower bound on $y_{j}$ is given by

$$
y_{j} \geqslant \max \left(a_{j}, c_{j}\right)
$$

where

$$
\begin{aligned}
& c_{j}=\sum_{p=1}^{j-1} \ell(T, p, j)+\sum_{p=1}^{j-1} \sum_{q=1}^{p} \max [\ell(U, p, q, j) \cdot \ell(V, p, q, j), \ell(U, p, q, j) \cdot u(V, p, q, j), \\
& u(U, p, q, j) \cdot \ell(V, p, q, j), u(U, p, q, j) \cdot u(V, p, q, j)\}
\end{aligned}
$$


Consider the separable problem created in section 3 . Since $y 3=y_{1}+y_{2}$ and the bounds on both $y_{1}$ and $y_{2}$ are 0 and 10 ,

$$
0<y_{3}<20 \text {. }
$$

(Recall $y_{4}$ was eliminated from the problem as unnecessary). Now

$$
z_{1,2}^{5,1}=\left[\sin \left(y_{1}\right)+\exp \left(-.5 y_{2}\right)\right] / 2
$$

and

$$
\mathrm{z}_{1,2}^{5,1}=\left[\sin \left(y_{1}\right)-\exp \left(-.5 y_{2}\right)\right] / 2
$$

Since

$$
-1=\inf \sin \left(y_{1}\right)<\sin \left(y_{1}\right)<\sup \sin \left(y_{1}\right)=1
$$

where

$$
0<y_{1} \leqslant 10
$$

and

$$
0=\text { inf } \exp \left(-.5 y_{2}\right)<\exp \left(-.5 y_{2}\right)<\sup \exp \left(-.5 y_{2}\right)<1 \text { where } 0 \leqslant y_{2} \leqslant 10 \text {, }
$$

it follows that the bounds on these new variables are

$$
\begin{array}{r}
-.5<z_{1,2}^{5,1}<1, \\
-1 .<z_{1,2}^{5,2}<.5 .
\end{array}
$$




\section{Computer Solution To Simple Problem}

The computer program which implements Falk's branch and bound procedure (see section 1) is called NUGLOBAL and is available from the Operations Research Division of the National Bureau of Standards. It uses a linear programming package called SEXOP written by Dr. Roy E. Marsten.

The user is required to write a FORTRAN subroutine (GETPHI) which supplies the values of the nonlinear functions defining the separable optimization problem. Input to this routine is the constraint index $i$, variable index $j$, and variable value $x_{j}$. The output is $F_{i j}\left(x_{j}\right)$. For the simple two variable example the SUBROUTINE GETPHI is listed in Appendix A.

The user is also required to supply a data file. The one used for this example is given in Appendix A. (This corresponds to the Run 3 to be discussed next.)

Computer instructions on how to use the NUGLOBAL system are in [Hoffman 1975].

For notational convenience and to conform to the separable format, problem (3.2) is rewritten

$$
\begin{aligned}
& \min \operatorname{ERF}\left(x_{3}\right)+\left(x_{4}\right)^{2}-\left(x_{5}\right)^{2} \\
& \left(x_{1}, \ldots, x_{5}\right)
\end{aligned}
$$


Sub ject to:

$$
\begin{aligned}
& x_{1}+x_{2}-x_{3}=0 \\
& -\sin \left(x_{1}\right)+x_{4}+x_{5}=0 \\
& -\exp \left(-.5 x_{2}\right)+x_{4}-x_{5}=0 \\
& -x_{3} \quad<-10 \\
& 0 \leqslant x_{1}<10, \\
& 0<x_{2}<10, \\
& 0<x_{3}<20, \\
& -.5 \leqslant x_{4}<1, \\
& 1<x_{5}<.5 .
\end{aligned}
$$

For "Run $1 "$ the first variable was "divided into nine variables", i.e. $\mathrm{P}_{1}=8$ (see Section 10). That is, in the linear programming problem $\theta_{1,0}, \ldots, \theta_{1,8}$ "represented" $x_{1}$. Variables $x_{2} \cdot x_{3} \cdot x_{4} \cdot x_{5}$ were replaced by 6 variables each. The resulting linear programming problems had $33 \theta$ variables.

To get the global minimizer to problem (P) required the solution of fifteen linear programing problems. The solution obtained was

$$
x^{*}=(5.0,0.0,5.0,0.020538,-0.97946)
$$

The value of the piece-wise linear approximation to the objective function was $(.052861)$. The value of the true objective function at this point is $(0.04107572)$.

The second run ("Run 2") used $p_{j}=10, j=1, \ldots, 5$. This created linear programming problems with $55 \Theta$ variables. This required the solution of 12 linear programming problems to obtain the global solutions to Problem (P). 
The solution obtained was the same as run $1, x^{*}=(5.0,0.0,5.0,0.020538$, $-0.97946)$. The value of the piece-wise linear objective function was $(0.04402)$. The true objective function value there is, as before, $(0.04107)$.

These two values are closer than in run 1 because of the finer grid approximation in Run 2 .

In Run 3, $p_{j}=25, j=1, \ldots, 5$. Each $x_{j}$ was represented by $26 \Theta$ variables. Each linear programming problem had 130 variables. Nine linear programs were required to find the solution $x^{*}=(4.8,0.0,4.8,0.0019177,-0.99808)$. The true objective function value there is $(0.00383)$ with piece-wise linear approximate value of $(0.00455)$. These results are summarized in Table 1 .

\begin{tabular}{|c|c|c|c|c|c|c|}
\hline $\begin{array}{c}\text { \# of LP } \\
\text { Vars. }\end{array}$ & $\begin{array}{c}\text { LPS } \\
\text { Solved }\end{array}$ & $x_{1}$ & $x_{2}$ & $x_{3}$ & $x_{4}$ & $x_{5}$ \\
\hline Run 1 33 & 15 & 5.0 & 0.0 & 5.0 & .0205 & -0.9795 \\
\hline Run 2 55 & 12 & 5.0 & 0.0 & 5.0 & 10205 & -0.9795 \\
\hline Run 3.130 & 9 & 4.8 & 0.0 & 4.8 & .0019 & -0.9981 \\
\hline $\begin{array}{c}\text { Theoretica1 } \\
\text { Solution }\end{array}$ & $\begin{array}{c}4.71 \\
=3 \pi / 2\end{array}$ & 0.0 & 4.71 & 0.0 & -1.0 \\
\hline
\end{tabular}

Table 1 - Computer Effort Required to Solve LP Approximating Problems 


\section{Direct Solution of Simple Problem}

Another approach for finding global minimizers to nonconvex programming problems (see [McCormick 1976] for more details) is to solve a sequence of "convex underestimating problems." Convex programming problems are problems which can be written:

$$
\begin{gathered}
\text { minimize } f(x) \\
\text { subject to } \\
g_{i}(x) \geqslant 0, i=1, \ldots, m
\end{gathered}
$$

where the functions

$$
\text { f, }\left\{-g_{i}\right\}_{i=1}^{m}
$$

are convex functions.

The nice property of convex programming problems is that local minimizers are global minimizers. The isovalue contours resemble ellipses, parabolas, or lines. It is clear from Figure 2 that the objective function of problem (2.2) is not a convex function.

The constraints of a convex programming problem define a convex set for the feasible region. For this simple problem, the constraint $\left(x_{1}+x_{2}\right)^{2}>10$ does not define a convex set. However, the intersection of the set of points satisfying this with the feasible region is a convex set.

A convex underestimating problem for a general problem is one where the objective function is convex and underestimates the given objective function in the feasible region and where the constraint region is a convex set and 
contains the original feasible region. Presented in [McCormick 1976] is an

algorithm for finding a convex underestimating problem when the original optimization problem is factorable with bounded variables.

The key to implementing this approach is the ability to compute, for a function of a single variable in an interval, its convex envelope. This is the highest convex function which underestimates the function in the interval. Thus, for example, the convex envelope of $\sin \left(x_{1}\right)$ where $0 \leqslant x_{1} \leqslant 10$ is

$$
\begin{aligned}
& -0.21723 x_{1}, \quad 0<x_{1}<4.49342 \\
& \operatorname{vexsin}\left(x_{1}\right)=\quad \sin \left(x_{1}\right), \quad 4.49342 \leqslant x_{1} \leqslant 4.79946 \\
& \left(-1.41353+0.08695 x_{1}\right), 4.79945 \leqslant x_{1} \leqslant 10
\end{aligned}
$$

This is shown in Figure 3, where the notation vex $f(\cdot)$ is used. Proper notation would indicate the interval. Thus vexsin $\left[x_{1}, 0,10\right]$ should be used, but for notational simplicity this is not done.

$$
\begin{array}{lr}
-.21723 x & 0<x<4.493719 \\
\sin (x) & 4.493419<x<4.7994496 \\
-1.4135281 & 4.7994496<x<10 \\
+.0869507 x &
\end{array}
$$



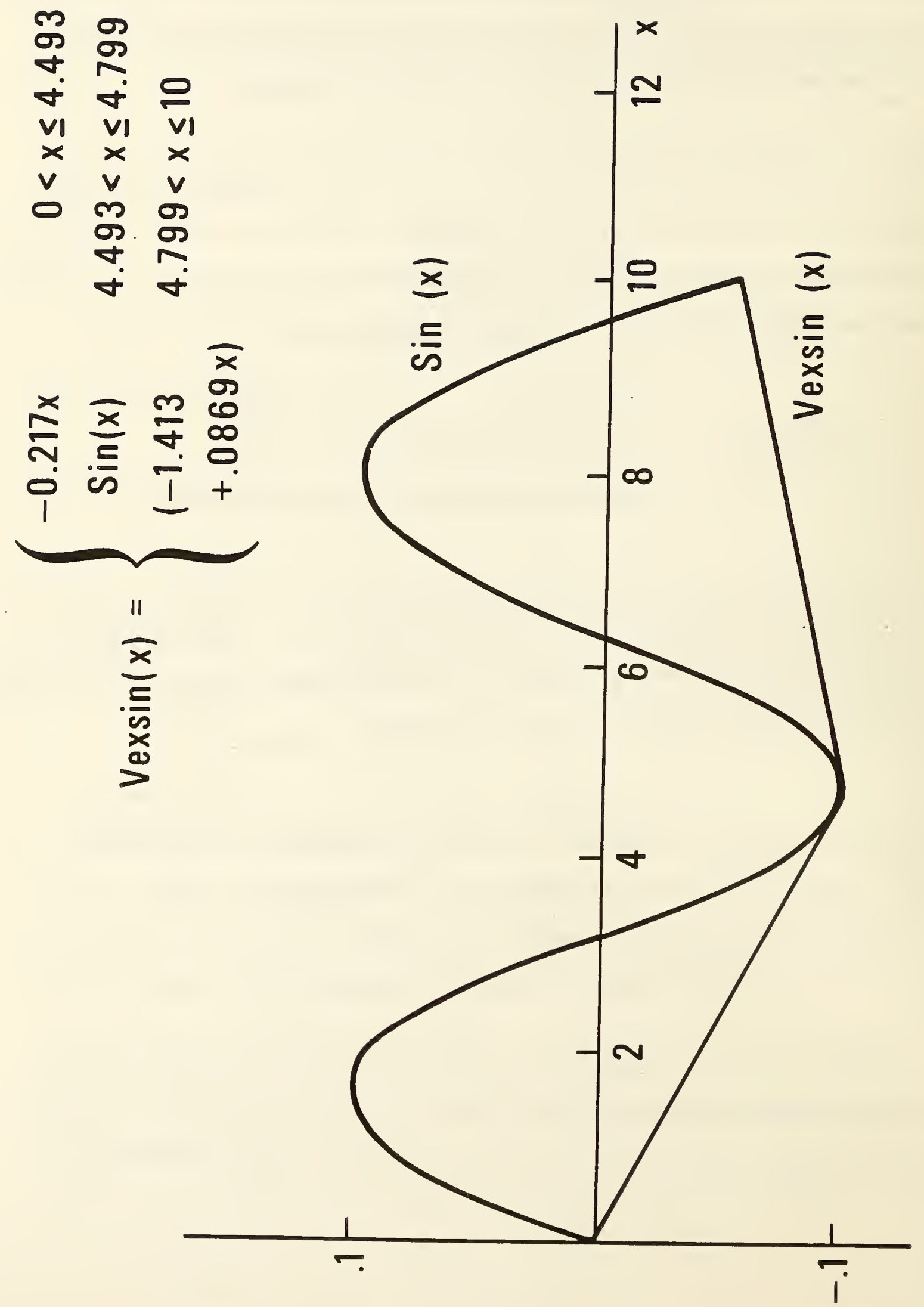

Figure 3. Convex Envelope of Sine Function $[0,10]$ 
Using the general techniques in the above referenced article, a convex underestimating function for the objective function of example problem (2.2) will be constructed.

Since $0 \leqslant x_{1}<10$ and $0 \leqslant x_{2}<10$, then $0 \leqslant x_{1}+x_{2}<20$. Consider

$$
\phi\left(x_{1}+x_{2}\right)=k \int_{0}^{x_{1}+x_{2}} \exp \left(-t^{2}\right) d t
$$

in this interval. It is clear from Figure 4, that .05 $\left(\mathrm{x}_{1}+\mathrm{x}_{2}\right)$ is a "high" convex underestimating function in this interval to a close approximation. 


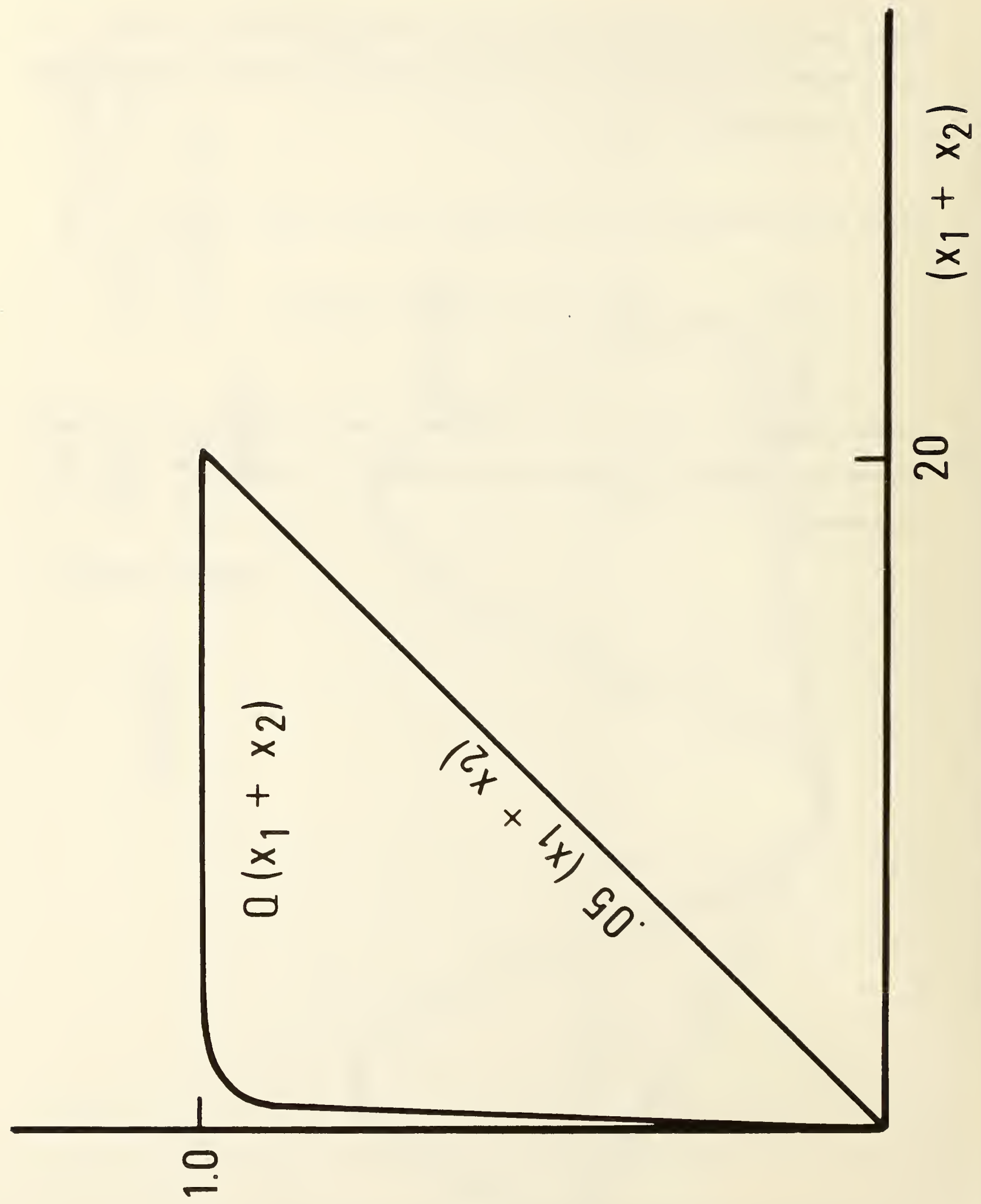

Figure 4. A Convex Underestimating Function 
Since $0<\mathrm{x}_{1}<10$, then $-1<\sin \left(\mathrm{x}_{1}\right)<1$. Since $0<\mathrm{x}_{2}<10$, then

$$
c_{1} \equiv \exp (-5) \leqslant\left(-.5 \mathrm{x}_{2}\right) \leqslant 1 \text {. }
$$

Thus,

$$
\left[\sin \left(x_{1}\right)+1\right]\left[\exp \left(-.5 x_{2}\right)-c_{1}\right]<0
$$

or

$$
\begin{aligned}
\sin \left(\mathrm{x}_{1}\right) \cdot \exp \left(-.5 \mathrm{x}_{2}\right) & >-\exp \left(-.5 \mathrm{x}_{2}\right)+c_{1} \sin \left(\mathrm{x}_{1}\right)+c_{1} \\
& >-1+c_{2} \mathrm{x}_{2}+c_{1}\left[\operatorname{vexsin}\left(\mathrm{x}_{1}\right)\right]+c_{1}
\end{aligned}
$$

where

$$
c_{2}=\left(-c_{1}+1\right) / 10=.0993262 .
$$

Note that $-1+.099326 \mathrm{x}_{2}$ is the convex envelope of $-\exp \left(-.5 \mathrm{x}_{2}\right)$ in the interval $[0,10]$.

Also,

$$
\left[\sin \left(c_{1}\right)-1\right]\left[\exp \left(-.5 x_{2}\right)-1\right] \geqslant 0
$$

or

$$
\begin{aligned}
\sin \left(x_{1}\right) \cdot \exp \left(-.5 x_{2}\right) & \geqslant \exp \left(-.5 x_{2}\right)+\sin \left(x_{1}\right)-1 \\
& >\exp \left(-.5 x_{2}\right)+\operatorname{vexsin}\left(x_{1}\right)-1 .
\end{aligned}
$$

Since the maximum of two convex functions is convex, a convex underestimating function for the product takes the form (combining (6.2) and (6.3)) $\sin \left(x_{1}\right) \cdot \exp \left(-.5 x_{2}\right) \geqslant \max \left[c_{1}-1+c_{2} x_{2}+c_{1} \operatorname{vexsin}\left(x_{1}\right), \exp \left(-5 x_{2}\right)+\operatorname{vexsin}\left(x_{1}\right)-1\right]$.

Using this with (6.1) gives the full convex underestimating function:

$$
k \int_{0}^{x_{1}+x_{2}} \exp \left(-t^{2}\right) d t+\sin \left(x_{1}\right) \cdot \exp \left(-.5 x_{2}\right)
$$

$\geq .05\left(x_{1}+x_{2}\right)+\max \left[c_{1}-1+c_{2} x_{2}+c_{1} \operatorname{vexsin}\left(x_{1}\right), \exp \left(-.5 x_{2}\right)+\operatorname{vexsin}\left(x_{1}\right)-1\right]$.

Isovalue contours of this function are plotted in Figure 5. 
The global minimizer for function (6.5) in the rectangle $0 \leqslant x 1 \leqslant 10$, $0 \leqslant x_{2} \leqslant 10$ is $(4.6623681,0$.$) . The first component is a solution of the$ equation arccos $\left(x_{1}\right)=-.05$. This is to be compared with the true solution of the original problem $(4.712389,0$.$) .$

Development of the convex underestimating function above took no advantage of the restriction that

$$
-\left(x_{1}+x_{2}\right)^{2}<-10
$$

must hold. A convex underestimating function of $-\left(x_{1}+x_{2}\right)^{2}$ in the rectangle

$$
0 \leqslant x_{i} \leqslant 10, i=1,2
$$

is

$$
-20\left(x_{1}+x_{2}\right)<-\left(x_{1}+x_{2}\right)^{2} \text {. }
$$

Thus the constraint can be imposed

or

$$
-20\left(x_{1}+x_{2}\right)<-10
$$

$$
x_{1}+x_{2}>.5
$$

The convex underestimating function of the objective function of (2.2) can now be restricted to the region where $.5 \leqslant\left(x_{1}+x_{2}\right) \leqslant 20$. This gives the tighter convex underestimating function

$$
.024593\left(x_{1}+x_{2}\right)+.5091315
$$

Using this with (6.4) gives a tighter convex underestimating function and the resulting solution of this problem is $(4.687793,0$.$) . The first component is$ a solution of $\arccos \left(x_{1}\right)=-.024593$. 


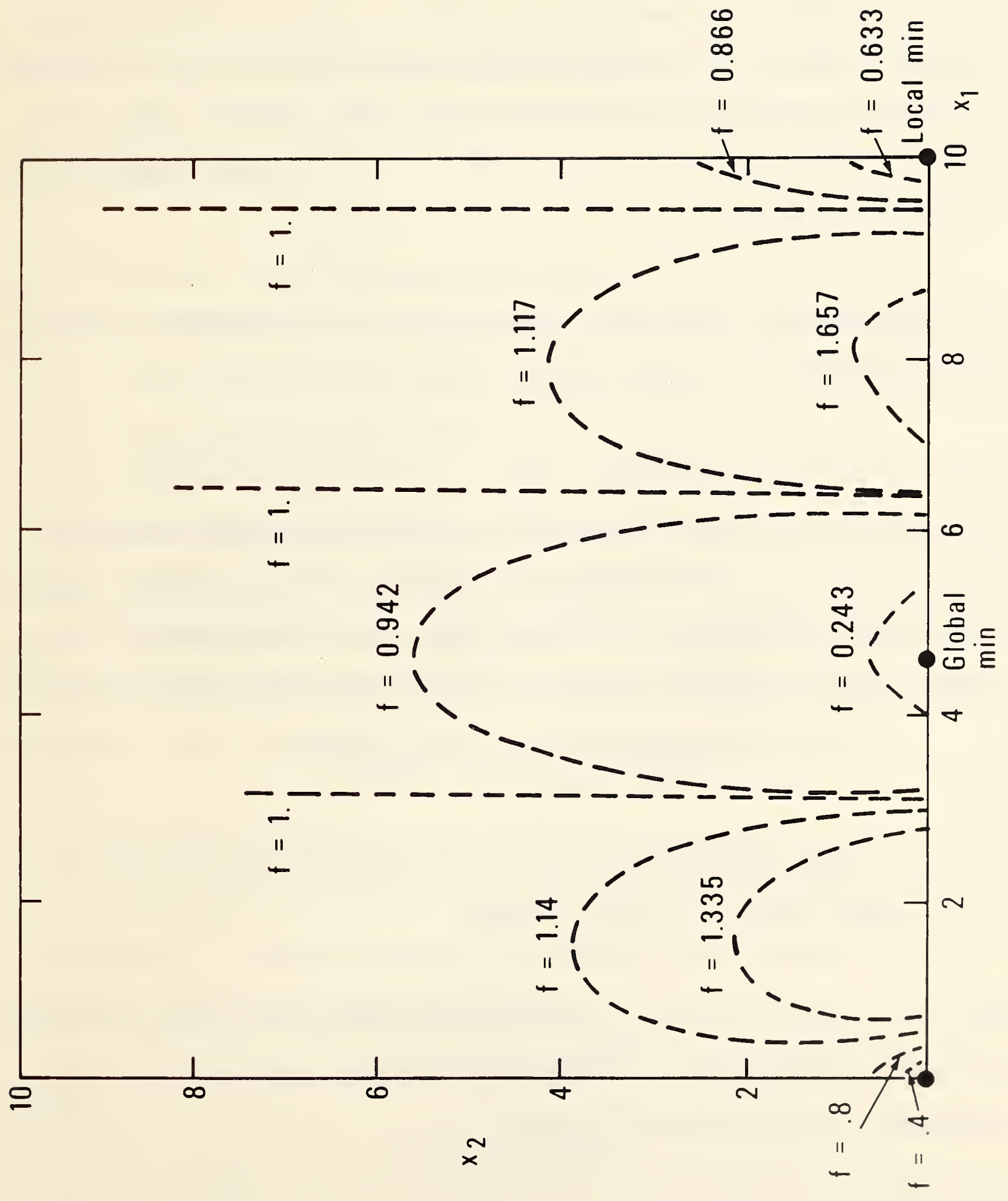

Figure 5. Isovalue Contours of Convex Underestimating Function 


\section{Application To Nonlinear Least Squares}

An important source of nonlinear programming problems is the area of parameter estimation. In particular the finding of the "best" parameter values which define a functional form often gives rise to an unconstrained, nonconvex optimization problem.

Let $\mathrm{x}$ be a vector of "independent" variables, and $\mathrm{y}$ a "dependent" variable which is thought to be related to $\mathrm{x}$ via the equation

$$
y=f(x, E) \text {, }
$$

where $\mathrm{E}$ is a vector of parameters and $\mathrm{f}$ is a predetermined functional form. Let $\left\{y_{i}\right\}, i=1, \ldots, r$ be observations of $y$ simultaneous with observations $\left\{x_{i}\right\}, i=1, \ldots, r$ of the independent variables. There is a body of theory (See [Goldfeld and Quandt 1972]) which states, under the appropriate assumptions, that the "best" values of $\left\{E_{j}\right\}$ are those which solve the problem

$$
\underset{E}{\operatorname{minimize}} \sum_{i=1}^{r}\left[y_{i}-f\left(x_{i}, E\right)\right]^{2} .
$$

In the reference above is also a theory of the probability distribution of the point estimate given by the above problem.

When $\mathrm{f}$ is linear in $\mathrm{E}$, this is the well-known linear least squares problen and the function minimized is a positive (semi) definite quadratic form. Local ninimizers of this are global minimizers. 
When $f$ is nonlinear in the parameters $\left\{E_{j}\right\}$ the problem is much more complicated and the possibility that local, but not truly global solutions of that problem exist must be considered. When the $E_{j}$ 's are physical constants to be estimated, as in many scientific applications, this difficulty has serious implications.

In this section a small parameter estimation problem is considered. The general separation method given in Section 3 plus some ad hoc techniques are used to create an equivalent separable problem. This is in turn solved by the solution algorithm of section 1 .

It should be noted that the number of variables in the separated problem is greater than or equal to the number of parameters to be estimated plus the number of observations $(r)$. If the function $f$ is complicated, more separation is required and this can give rise to a separable problem in a large number of variables with an accompanying large linear approximation problem.

In Figure 6 is plotted the fraction of college men married versus age. The data for the problem is in Table 2. It is thought that the dependent variable $y$ (fraction of college men married) is related to the independent variable $x$ (age in years) by the functional equation

$$
y=p \Phi[(x-\mu) / \sigma]
$$

where

$$
\Phi(z)=\int_{-\infty}^{y} \phi(t) d t,
$$

and

$$
\phi(t)=(2 \pi)^{-.5} \exp \left(-t^{2} / 2\right)
$$

(The normal density function.) 
The parameters, whose best values are to be estimated from the data of Table 2 are $p$ (the ultimate fraction of these married), $\mu$ (the mean of the distribution) and $\sigma$ (the standard deviation).

\begin{tabular}{|c|ccccccc|}
\hline$i$ & 1 & 2 & 3 & 4 & 5 & 6 & 7 \\
\hline$x_{i}$ & 14.6 & 16.8 & 18.7 & 20.6 & 23.1 & 27.1 & 32.0 \\
\hline$y_{i}$ & 0.000 & 0.004 & 0.015 & 0.075 & 0.315 & 0.571 & 0.737 \\
\hline
\end{tabular}

Table 2

Data for Example 


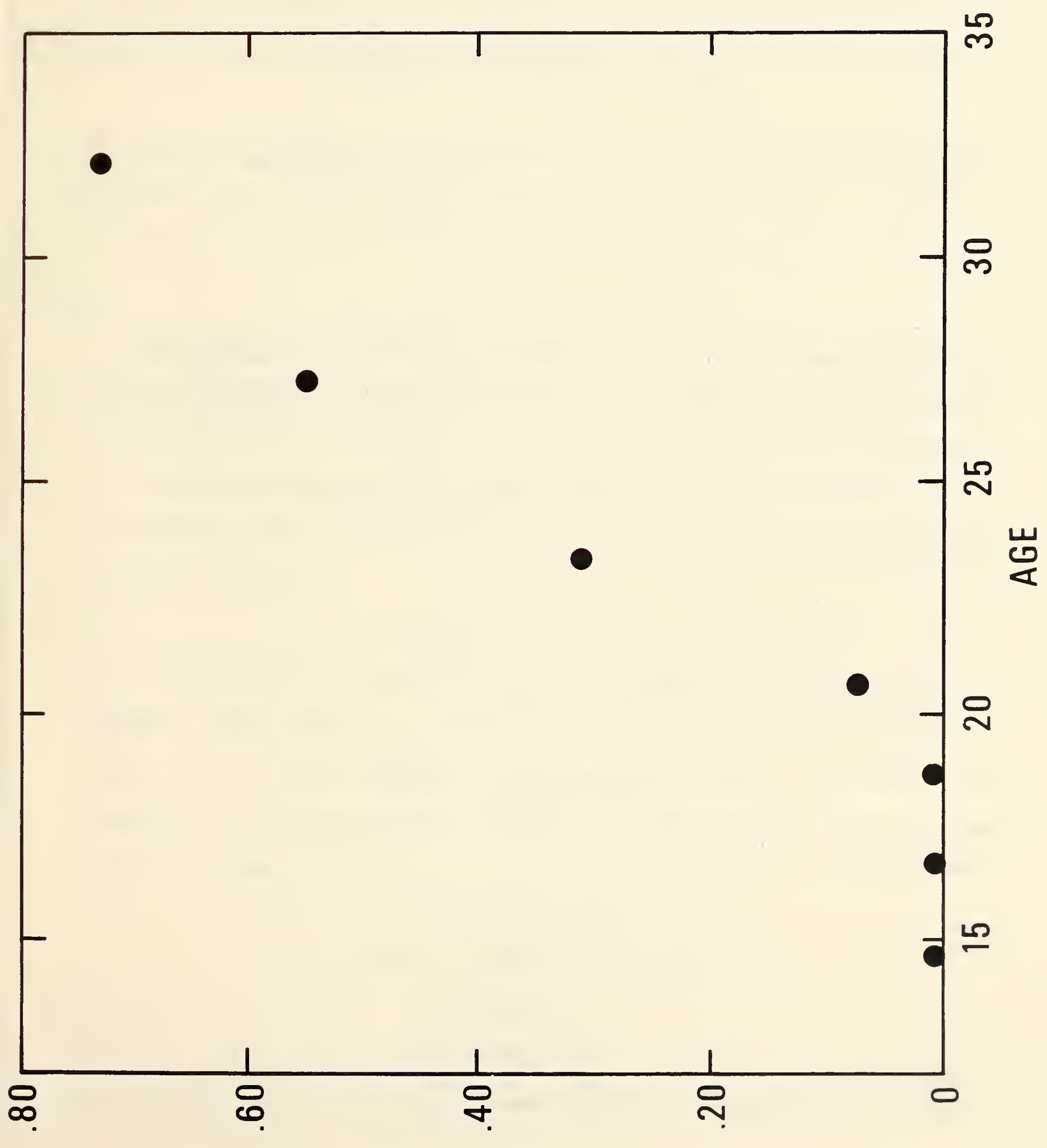

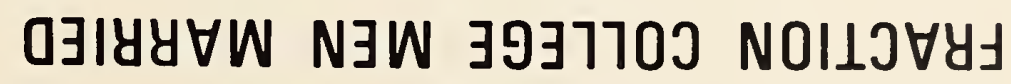

Figure 6. Plotted Data for Example 
The optimization problem to be solved is

$$
\min _{(\mu, \delta, p)} \sum_{i=1}^{7}\left\{y_{1}-p \Phi\left(\left[x_{1}-\mu\right] / \sigma\right)\right\}^{2}
$$

As a first step in converting this to a separable problem set

$$
v_{1}=p \Phi\left[\left(x_{1}-\mu\right) / \sigma\right], i=1, \ldots, 7,
$$

and

$$
z_{i}=\left(x_{i}-\mu\right) / \sigma \quad, i=1, \ldots, 7 \text {. }
$$

A trick not mentioned in section 3 but one which is often useful is to transform a product into a sum by taking logarithins. Doing this to (7.2) yields

$$
\ln v_{1}=\ln p+\ln \Phi(z i), i=1, \ldots, 7
$$

By setting

$$
\begin{gathered}
\mu=\omega_{1}+\omega_{2}, \\
1 / \sigma=\omega_{1}-\omega_{2},
\end{gathered}
$$

the original nonseparable problem becomes

$$
\operatorname{minimize} \sum_{i=1}^{7}\left(y_{1}-v_{1}\right)^{2}
$$

sub ject to

$$
\begin{aligned}
1_{n} p-\ln v_{1}+\ln \Phi\left(z_{1}\right) & =0, i=1, \ldots, 7 \\
x_{1}\left(\sigma^{-1}\right)-z_{1}-\left(\omega_{1}\right)^{2}+\left(\omega_{2}\right)^{2} & =0, i=1, \ldots, 7 \\
\mu-\omega_{1}-\omega_{2} & =0 \\
\sigma^{-1}-\omega_{1}+\omega_{2} & =0
\end{aligned}
$$

where the minimization is performed over $\left(\mu, 1 / \sigma, 1 \mathrm{n},\left\{\mathrm{v}_{1}\right\},\left\{z_{1}\right\}, \omega_{1}, \omega_{2}\right)$. 
Note that the separated problem contains $1 / \sigma$ and $\ln p$ instead of $\sigma$ and $p$. This was done because the new formulation is equivalent and is linear in the transformed parameters (which are variables of the optimization problem).

From the physical model it is obvious that the parameters are constrained as $\mu>0, \sigma>0,0<p<1$. If the data is consistent with the model it is expected that these constraints are implicit and will not be binding at the solution.

One requirement of the global minimization technique outlined in Section 1 is that lower and upper bounds are required on the problem variables. It should be pointed out that this can be an advantage since often, from knowledge of the problem, good bounds are available. This can reduce the region in which optimization takes place and thus cut down on the amount of computer effort required to solve it.

From the data, obvious bounds on the original three parameters are: $18<\mu<30,1<\sigma<10$, and $.5<p<1$. The techniques of Section 4 are then used to place bounds on the new variables of the separated problem. The names of the variables of the separated problem, their $x_{j}$ indices and their upper and lower bounds are given in Table 3 . 


\begin{tabular}{|c|c|c|c|c|c|}
\hline $\begin{array}{c}\text { Variable } \\
\text { Index } \\
\quad j\end{array}$ & $\begin{array}{c}\text { Variable } \\
\text { Name }\end{array}$ & $\begin{array}{l}\text { Lower } \\
\text { Bound }\end{array}$ & $\begin{array}{l}\text { Upper } \\
\text { Bound }\end{array}$ & $\begin{array}{l}\text { Cuts } \\
\text { Run } 1\end{array}$ & $\begin{array}{l}\text { Cuts } \\
\text { Run } 2\end{array}$ \\
\hline 1 & $\mu$ & 18. & 30. & 1 & 1 \\
\hline 2 & $1 / \sigma$ & .1 & 1. & 1 & 1 \\
\hline 3 & $\ln p$ & -.693 & 0 & 1 & 1 \\
\hline 4 & $\mathrm{v}_{1}$ & .0006 & .37 & 5 & 5 \\
\hline 5 & $\mathrm{v}_{2}$ & .0006 & .45 & 5 & 5 \\
\hline 6 & $v_{3}$ & .0006 & .76 & 5 & 5 \\
\hline 7 & $v_{4}$ & .0006 & 1. & 5 & 5 \\
\hline 8 & $\mathrm{v}_{5}$ & .0006 & 1. & 5 & 5 \\
\hline 9 & $v_{6}$ & .0009 & 1. & 5 & 5 \\
\hline 10 & $v_{7}$ & .280 & 1. & 5 & 5 \\
\hline 11 & $z_{1}$ & $(-3)$. & -.34 & 3 & 5 \\
\hline 12 & $z_{2}$ & -3 & -.12 & 3 & 5 \\
\hline 13 & $z_{3}$ & -3 & 0.7 & 3 & 5 \\
\hline 14 & $z_{4}$ & -3 & 2.6 & 3 & 5 \\
\hline 15 & $z_{5}$ & -3 & 3. & 3 & 5 \\
\hline 16 & $z_{6}$ & -2.9 & 3. & 3 & 5 \\
\hline 17 & $z_{7}$ & .2 & 3. & 3 & 5 \\
\hline 18 & $\omega$ & 8.0 & 16. & 5 & 5 \\
\hline 19 & $\omega$ & 8.0 & 16. & 5 & 5 \\
\hline
\end{tabular}

Table 3: Data for Least Squares Problem in Separable Form 
A modified Newton method was applied directly to problem (7.1) and gave the solution

$$
\mu=24.4, \sigma=3.28, p=0.73
$$

Two computer runs were made on the separated problem. The data files and subroutine are in Appendix B.

The number of cuts for each of the nineteen variables are given in Table 3 . The number of $\theta$-variables in the linear programming problems associated with each of the variables in the separated problem is equal to the number of cuts plus one. Thus in Run 1 each linear programming problem had 88 variables, and in Run 2 each had 102 variables. The lower bound for $z_{1}$ in Run 1 was -3 and it was -5 in Run 2 .

For Run 1 the approximate solution was obtained after the solution of 41 linear programming poblems and was

$$
\mu=22.19, \sigma=4.28 \text {, and } \mathrm{p}=.57
$$

For Run 2 the approximate solution was obtained after the solution of 62 linear programming problems with values

$$
\mu=22.54, \sigma=3.31 \text {, and } p=.72 \text {. }
$$

These results are summarized in Table 4 . 


\begin{tabular}{|c|c|c|c|c|c|}
\hline Method & $\mu$ & $\sigma$ & $p$ & $\begin{array}{c}\text { \# of } \\
\text { Variables }\end{array}$ & $\begin{array}{c}\text { MP Problems } \\
\text { Solved }\end{array}$ \\
\hline Newton & 24.14 & 3.28 & 0.73 & 3 & 1 (NLP) \\
\hline Run 1 & 22.19 & 4.28 & 0.57 & 88 & 41 (LP) \\
\hline Run 2 & 22.54 & 3.31 & 0.72 & 102 & 62 (LP) \\
\hline
\end{tabular}

Table 4: Data Relating to Solution of of Least Squares Problem 


\section{Discussion of Results and Areas of Future Research}

In this paper it was shown how general nonlinear programming problems can automatically be converted to equivalent separable problems when they consist of factorable functions. Global solutions to linear approximations of these separable programs are then obtained using a branch and bound technique with linear programming subproblems. Two small examples were solved this way illustrating the feasibility of the approach.

First, experiments should be made on the placing of the points which determine the piece-wise linear approximations to the nonlinear functions of a single variable. In solving example (3.1), refinement of the grid from Run 1 to Run 2 did not result in any improvement in the solution approximation (see Table 1). The final answer obtained (Run 3) only agreed with the correct solution to one significant place even though the number of 0 variables approximating the nonlinear functions was increased significantly. The reason for this was that the grid refinement strategy used allowed only for equally spaced intervals. Judicious placement should have as a result better accuracy and fewer 0-variables.

Recent efforts in this area are encouraging. In [Grotzinger 1981] problem (1.2) was solved to the accuracy $(4.20,0$.$) requiring 11$ linear programming problems none of which had more than 25 0-variables. 
The second area of improvement is to lower the effort required to solve the linear programming problems by using the inverse matrix (basis) information available from similar LP's solved by the branch and bound procedure. This should considerably reduce the computation time. No advantage of available partial basis information was used in solving the problems.

Advantage can also be taken of this information when new variables are added to improve the linear approximation.

Until efficient nonlinear programming codes are available, using linear programming codes as subroutines to solve general nonlinear programming problems seems a reasonable approach and one which could be implemented on a production basis without much additional effort. 


\section{Ref erences}

1. Beale, E.M.L., and John Tomlin, Special Facilities in a General Mathematical Programming System for Nonconvex Problems Using Ordered Sets of Variables in ( $\mathrm{J}$. Lawrence ed.) Proc. of the Fifth International Conference on Operations Research (1970) pp. 447-454, Tavistock Publication, London.

2. Falk, James E. and Richard M. Soland, An Algorithm for Separable Nonconvex Programming Problems, Man. Sc. 15 (1969) pp. 550-569.

3. Falk, J.E., An Algorithm for Locating Approximate Global Solutions of Nonconvex, Separable Problems, Technical Paper Serial T-262, Institute for Management Science and Engineering, the George Washington University, Washington, DC, April 1972 .

4. Ghaemi, Abofazl, and Garth P. McCormick, Symbolic factorable SUMT: What is it? How is it used?, Technical Paper Serial T-402, Institute for Management Science and Engineering, The George Washington University, Washington, DC, May 1979.

5. Goldfeld, Stephen M., and Richard E. Quandt, Nonlinear Methods in Econometrics North-Holland, Amsterdam, 1972.

6. Grotte, Jeffrey H., A Computer Program for Solving Separable Nonconvex Optimization Problems, IDA Paper P-1318, Institute for Defense Analysis, Arlington, Virginia, January 1978.

7. Grotzinger, Stephen J., Personal Communication.

8. Hoffman, Karla L., NUGLOBAL--USER'S GUIDE, Technical Memorandum Serial TM-64866, Institute for Management Science and Engineering, The George Washington University, March 1975. 
9. Hoffman, Karla L., A Method for Globally Minimizing Concave Functions Over Convex Sets, Math. Prog. 20 (1981), pp. 22-32.

10. Mancini, Louis J. and Garth P. McCormick, Bounding Global Minima, Math. Oper. Res. 1 (1976) pp. 50-53.

11. Mancini, Louis J. and Garth P. McCormick, Bounding Global Minima with Interval Arithmetic, Operations Research 27 (1979) pp. 743-754.

12. McCormick, Garth P., Attempts to Calculate Global Solutions of Problems that May Have Local Minima, in (F.A. Lootsma, ed.) Numerical Methods for Nonlinear Optimization, 1972, Academic Press, New York, pp. 209-221.

13. McCormick, Garth P., Converting General Nonlinear Programming Problems to Separable Nonlinear Programming Problems, Technical Paper Serial T-267, Institute for Management Science and Engineering, The George Washington University, Washington, DC, June 1972 .

14. McCormick, Garth P., Optima1 Design of a Corrugated Transverse Bulkhead; An Example of the Use of the Factorable Programming Language, Technical Paper Serial T-313, Institute for Managements Science and Engineering, The George Wahington University, Washington, DC, 1975.

15. McCormick, Garth P., Computability of Global Solutions to Factorable Nonconvex Programs: Part I--Convex Underestimating Problems, Math. Prog. 10 (1976) pp. 147-175.

16. McCormick, Garth P., Locating An Isolated Global Minimizer of a Constrained Nonconvex Program, Math. of Oper. Res., 5(1980) pp. $435-443$.

17. Soland, Richard M., An Algorithm for Separable Nonconvex Programming Problems II: Nonconvex Constraints, Man. Sc. 17 (1971) pp. 759-773. 
NBS.114A IREV. 2.80 )

U.3. DEPT. OF COMM.

1. PUBLICATION OR

2. Performing Organ. Report Nof 3. Publication Date

BIBLIOGRAPHIC DATA REPORT NO.

SHEET (See instructions)

NBSIR 85-3206

JULY 1985

4. TITLE AND SUBTITLE

Global Solutions to Factorable Nonlinear Optimization Problems Using Separable Programming Techniques

5. $A \cup T H O R(S)$

Garth P. McCormick

6. PERFORMING ORGANIZATION (If joint or other thon NBS, see instructions)

NATIONAL BUREAU OF STANDARDS

DEPARTMENT OF COMMERCE

N00014-75-C-0611

WASHINGTON, D.C. 20234

9. SPONSORING ORGANIZATION NAME AND COMPLETE ADDRESS (Street, City. Stote, ZIP)

National. Bureau of Standards

Center for Applied Mathematics

Operations Research Division

Gatthersburg, MD 20899

10. SUPPLEMENTARY NOTES

Document describes a computer program: SF-185, FIPS Software Summary, is attached.

11. ABSTRACT (A 200-word or less foctual summary of most significont information. If document includes o significant bibliogrophy or literoture survey, mention it here)

Many algorithms for obtaining global solutions to nonconvex optimization problems have been proposed in recent years. The methods farthest along computationally are those for separable problems. These use linear programming codes to solve sequences of LP problems formed from piece-wise linear approximations to the nonlinear functional forms. For a large class of optimization problems, called factorable programming problems, it is possible to create equivalent separable problems. This is done at a cost: additonal variables and constraints. In this paper the procedure for creating the equivalent separable problems is outlined and a brief description is given of a global solution algorithm due to Falk. A small example is given illustrating the above techniques. The example is also solved using a more direct method. Application to the solution of nonlinear least squares is illustrated with another example. Discussion of areas of research for improving the efficiency of this approach concludes the paper.

12. KEY WORDS (Six to twelve entries; alphabetical order; copitalize only proper nomes; ond seporote key words by semicolons) branch and bound; factorable functions; mathematical programming; nonconvex programing; nonlinear least squares; nonlinear optimization; separable opt1mizat1on.

13. AVAILABILITY

X. Unlimited

For Official Distribution. Do Not Release to NTIS

Order From Superintendent of Documents, U.S. Government Printing Office, Washington. D.C. 20402.

14. NO. OF PRINTED PAGES

Order From National Technical Information Service (NTIS), Springfield, VA. 2216I 


